ARTICLE

Received 16 Jan 2016 | Accepted 27 Apr 2016 | Published 31 May 2016

DOI: $10.1038 /$ ncomms11772

OPEN

\title{
Wolbachia infections in natural Anopheles populations affect egg laying and negatively correlate with Plasmodium development
}

\author{
W. Robert Shaw ${ }^{1, \star}$, Perrine Marcenac ${ }^{1, \star}$, Lauren M. Childs ${ }^{2,3}$, Caroline O. Buckee $^{2,3}$, Francesco Baldini ${ }^{4}$, \\ Simon P. Sawadogo ${ }^{5}$, Roch K. Dabiré ${ }^{5}$, Abdoulaye Diabaté $^{5}$ \& Flaminia Catteruccia ${ }^{1}$
}

The maternally inherited alpha-proteobacterium Wolbachia has been proposed as a tool to block transmission of devastating mosquito-borne infectious diseases like dengue and malaria. Here we study the reproductive manipulations induced by a recently identified Wolbachia strain that stably infects natural mosquito populations of a major malaria vector, Anopheles coluzzii, in Burkina Faso. We determine that these infections significantly accelerate egg laying but do not induce cytoplasmic incompatibility or sex-ratio distortion, two parasitic reproductive phenotypes that facilitate the spread of other Wolbachia strains within insect hosts. Analysis of 221 blood-fed A. coluzzii females collected from houses shows a negative correlation between the presence of Plasmodium parasites and Wolbachia infection. A mathematical model incorporating these results predicts that infection with these endosymbionts may reduce malaria prevalence in human populations. These data suggest that Wolbachia may be an important player in malaria transmission dynamics in Sub-Saharan Africa.

\footnotetext{
${ }^{1}$ Department of Immunology and Infectious Diseases, Harvard T.H. Chan School of Public Health, Boston, Massachusetts 02115, USA. ${ }^{2}$ Center for Communicable Disease Dynamics, Harvard T.H. Chan School of Public Health, Boston, Massachusetts 02115, USA. ${ }^{3}$ Department of Epidemiology, Harvard T.H. Chan School of Public Health, Boston, Massachusetts 02115, USA. ${ }^{4}$ Institute of Biodiversity, Animal Health and Comparative Medicine, University of Glasgow, Glasgow G12 8QQ, UK. ${ }^{5}$ Institut de Recherche en Sciences de la Santé/Centre Muraz, O1 BP 390 Bobo-Dioulasso 01, Burkina Faso.

* These authors contributed equally to this work. Correspondence and requests for materials should be addressed to F.C. (email: fcatter@hsph.harvard.edu).
} 
E very year nearly 200 million people contract malaria and around 450,000 people die from the disease, mostly young children in Sub-Saharan Africa ${ }^{1}$. Malaria transmission depends on the complex ecological determinants that drive population dynamics of Anopheles mosquitoes, the vectors of human malaria parasites. Measures aimed at the mosquito vector have been the mainstay of malaria control strategies 1,2 and have achieved significant decreases in the global burden of this disease over the last decade, primarily attributable to the widespread use of long-lasting insecticide-treated nets (LLINs) ${ }^{3}$. However, insecticide-based prevention tools are severely threatened by the rapid spread and global distribution of insecticide resistance in anopheline populations ${ }^{4,5}$, making the development of new insecticide-free tools for reducing malaria transmission a crucial priority 6 .

The use of Wolbachia bacteria-intracellular endosymbionts of arthropods and nematodes-has long been suggested for the control of mosquito populations transmitting viral or parasitic pathogens such as dengue fever and malaria, given their profound effects on both insect physiology and pathogen development ${ }^{7}$. These bacteria are best known as active modulators of host reproduction via the induction of mechanisms that promote their rapid invasion of insect host populations. The most commonly observed of these phenotypes is cytoplasmic incompatibility (CI), which prevents uninfected females from producing viable progeny after mating with infected males due to chromosomal segregation defects in the first cellular divisions of the fertilized egg (reviewed in ref. 8). However, infected females are able to produce viable progeny regardless of whether they mate with infected or uninfected males through unknown rescue mechanisms, an effect that favours Wolbachia's spread ${ }^{9,10}$. Additional phenotypes include vertical transmission from mother to progeny, and a female bias in the sex ratio through selective male killing, male feminization and female parthenogenesis (where unfertilized eggs develop as females) ${ }^{11-13}$. Combined with these properties, Wolbachia infections in many mosquito species can prevent pathogen development and block disease transmission from vector to human. The list of vectorborne pathogens affected by Wolbachia is broad and includes viral pathogens causing dengue, yellow fever, West Nile and Chikungunya, as well as Plasmodium parasites ${ }^{14-18}$, suggesting a general mechanism of pathogen blocking by an upregulated immune response in the mosquito host ${ }^{19-21}$, although not in all cases $^{16,22}$.

We have recently identified stable Wolbachia infections in natural populations of two important malaria vectors, Anopheles gambiae and Anopheles coluzzii, in Burkina Faso ${ }^{23}$, a country with a high malaria burden. Owing to several negative reports $^{24-26}$, it was previously believed that these bacteria were unable to infect Anopheles species, as also suggested by the difficulties to generate stably transinfected Anopheles lines in the laboratory (only recently surmounted ${ }^{14}$ ). Consequently, blocking of Plasmodium parasites was initially demonstrated using either transient injections of non-native Wolbachia strains into adult Anopheles females ${ }^{15,19}$, or a bird model of malaria infection transmitted by Aedes mosquitoes ${ }^{16}$. Our previous study showed that Wolbachia were transmitted from females to progeny with an average transmission frequency of $68 \%$ (ranging from 56 to $100 \%)$ and determined that the strain identified in Anopheles (which we named $w$ Anga) belongs to a new arthropod-specific supergroup $^{23}$. Wolbachia infections have since been confirmed in a subsequent study of $A$. coluzzii mosquitoes from the same region of Burkina Faso ${ }^{27}$.

As Wolbachia can profoundly perturb insect ecology, behaviour and physiology, and can significantly reduce transmission of some human pathogens, our previous findings provide an unprecedented opportunity to assess the physiological and reproductive impacts of $w$ Anga infection on anophelines and to determine how these effects influence the dynamics of malaria transmission. Here we explore the complex relationships between Anopheles mosquitoes, Plasmodium parasites and Wolbachia endosymbionts in natural populations. We find that $w$ Anga infections are persistent in $A$. coluzzii over several years, proving these bacteria are stable residents in these mosquito populations, and are also detected in another important malaria vector, Anopheles arabiensis. Although crosses between infected and uninfected individuals do not show evidence of CI, infected females lay eggs more rapidly than uninfected individuals. Sampling of natural populations shows that Wolbachia-positive females are infected with Plasmodium parasites at significantly lower frequencies than Wolbachia-negative individuals, unveiling a negative correlation between these endosymbionts and deadly malaria parasites. Modelling the effects of these endosymbionts on malaria transmission dynamics reveals that natural Wolbachia infections could have a significant impact on the prevalence of malaria in human populations in Sub-Saharan Africa.

\section{Results}

Wolbachia infections are stable and localize in the oocytes. We returned to Vallée du Kou near Bobo-Dioulasso, Burkina Faso, in 2014 to determine the prevalence of Wolbachia infections in A. coluzzii populations. We collected 602 mosquitoes and identified infections at a frequency of $46 \%$ (275/602). Furthermore, analysis of samples collected in 2013 in the town of Soumousso found wAnga at a frequency of $33 \%(16 / 49)$ in A. arabiensis (Supplementary Table 1), a species not effectively targeted by LLINs and indoor residual sprays as it primarily blood feeds and rests outdoors. The prevalence of $w$ Anga was moderate compared with that observed in naturally infected Aedes and Culex mosquitoes $^{24,28}$, although this may be due to difficulties in detection caused by low intensity of infection in some samples ${ }^{23}$. When considering all data since 2011, prevalence was variable between years (19-46\%) (Supplementary Table 1), suggesting physiological or ecological factors may influence Wolbachia dynamics over time.

With the aim to facilitate detection of these bacteria within the female, we established a stable $w$ Anga-infected mosquito line in our laboratory after colonization of A. coluzzii populations from the Vallée du Kou villages. We dissected ovaries from females and visualized $w$ Anga at a similar frequency (43\%) within the ovarian follicles by fluorescent in situ hybridization (FISH) experiments using a DNA probe that specifically hybridizes to Wolbachia $16 \mathrm{~S}$ nucleic acid sequences (Fig. 1a). In control experiments, no signal was detected in ovaries dissected from females treated with tetracycline to clear $w$ Anga infection (Fig. 1b), nor when using an excess of unlabelled probe of identical sequence in competition with the labelled probe (Fig. 1c).

wAnga does not induce CI but affects oviposition. Many Wolbachia strains induce CI when an infected male mates with an uninfected female, producing mostly inviable progeny. To determine whether natural Wolbachia infections induce CI and/or other reproductive phenotypes in field $A$. coluzzii, we collected eggs from blood-fed females in houses or larvae from breeding sites and, after adult emergence, performed crosses between virgin males and females using a force-mating technique, as field mosquitoes of this species do not mate in captivity. After mating, females were blood-fed and allowed to lay eggs individually in separate oviposition containers. We measured the number of eggs developed to assess the impact of infection on fecundity, and the larval hatch rates to measure effects on fertility and therefore detect a possible occurrence of CI. We also scored 

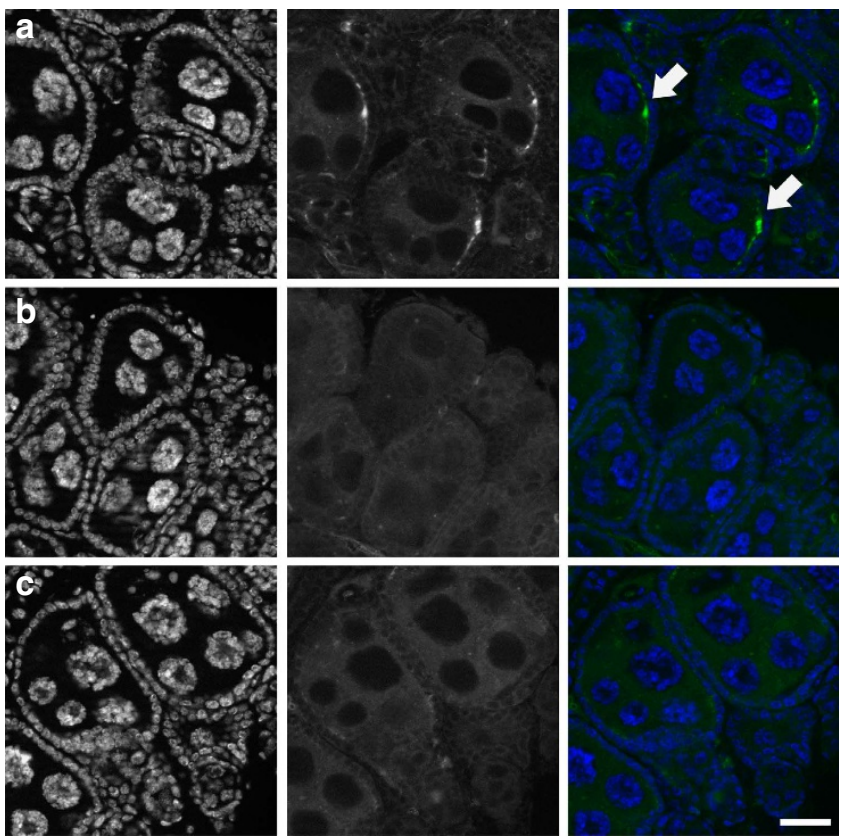

Figure 1 | wAnga localizes to the female germline. wAnga (in green, central panels) was visualized in the ovaries of 14- to 16-day-old A. coluzzii females by FISH. (a) wAnga is detected in ovarian follicles using a Cy3-labelled probe specific for 16S DNA (white arrows). (b,c) wAnga is absent from the follicles of tetracycline-treated control females (b) and in follicles of infected females in which the labelled probe was in competition with an identical unlabelled probe (1:20 labelled:unlabelled) (c). DNA is labelled with 4,6-diamidino-2-phenylindole (in blue, left panels). Scale bar, $20 \mu \mathrm{m}$.

the sex ratio of the progeny of each individual female by letting larvae grow to adulthood and counting males and females to determine whether wAnga induces sex distortions in these mosquitoes. Both parents of each brood were genotyped and screened for Wolbachia post hoc, and crosses between Wolbachiapositive males and Wolbachia-negative females (that is, those potentially inducing CI, shown in blue in Fig. 2) were compared with all other cross combinations (Wolbachia-positive males and females; Wolbachia-negative males and females; and Wolbachianegative males and Wolbachia-positive females). Although egg inviability was slightly higher in the crosses in which CI is predicted to occur, we did not observe any significant effect of $w$ Anga on egg inviability between our crosses, which suggests that this Wolbachia strain does not cause detectable CI in natural A. coluzzii populations (Kruskal-Wallis test, $\chi^{2}=1.25$, degrees of freedom (d.f.) $=3, P>0.05$; Fig. 2a). Moreover, we found no difference in the number of eggs developed (Kruskal-Wallis, $\chi^{2}=4.09$, d.f. $=3, P>0.05$; Fig. 2 b), eggs laid (Kruskal-Wallis, $\chi^{2}=0.686$, d.f. $=3, P>0.05$; Supplementary Fig. 1) or in the progeny sex ratio (Student's $t$-test on $\log _{10}$-transformed ratios, $t=0.257$, d.f. $=26, P>0.05$; Fig. 2 c).

We next assessed whether egg-laying behaviour is altered by $w$ Anga infection. To this end, blood-fed females from houses in the Vallée du Kou were placed in individual cups 2 days post blood feeding, and oviposition rates were counted daily. After assessing their infection status post hoc, we determined that $w$ Anga-infected females had laid eggs significantly more quickly than uninfected females (on average $0.73 \pm 0.11$ days, log-rank test, $\chi^{2}=32.36$, d.f. $=1, P<0.0001$; Fig. 2 d). This shortened oviposition timing could increase the number of gonotrophic cycles over the course of a female's lifespan, and suggests that wAnga-infected females may bite more frequently than uninfected individuals (Supplementary Fig. 2), with possible consequences for malaria transmission. However, as predicted by life history theory ${ }^{29}$, an increase in fecundity would likely induce a compensatory decrease in longevity (not measured in our experimental design), a key component for the completion of the parasite cycle within the mosquito vector.

wAnga interferes with natural Plasmodium infections. A crucial question concerning Wolbachia infections in Anopheles mosquitoes is whether these bacteria impact Plasmodium transmission by the mosquito vector. As Burkina Faso is a region of high malaria transmission, we reasoned that we could directly assess the effects of natural $w$ Anga infection on the prevalence of Plasmodium parasites in blood-fed females collected from the interior of houses in the Vallée du Kou. We dissected a total of 221 blood-fed A. coluzzii females (genotyped post hoc) 5 days post collection, a time when the blood meal is fully digested and oocyst development is underway. DNA was extracted from samples containing abdomens and thoraxes (encompassing the ovaries, the midgut and the salivary glands) dissected from individual females to unravel a possible interaction between the presence of Wolbachia and Plasmodium in these individuals. A total of 116 females were positive for $w$ Anga, with an infection prevalence of $52.5 \%(116 / 221)$. Plasmodium infections were detected in 12 females, producing a prevalence of $5.4 \%(12 / 221)$, comparable to previously reported data for this region ${ }^{30}$. We found a strong bias for Plasmodium infection in Wolbachia-negative individuals; 11 Plasmodium-positive females were negative for Wolbachia, and only 1 female showed co-infection with bacteria and parasites (Fisher's exact post hoc test on unnormalized data, two-tailed, $P=0.0018$; Fig. 3a and Supplementary Table 2). This highly significant reduction-over $90 \%$ - suggests that $w$ Anga may interfere with Plasmodium development in the mosquito vector, as shown in artificial Anopheles-Wolbachia combinations ${ }^{14,15,19}$

We went on to model whether natural Wolbachia infections would affect malaria transmission dynamics when these results, obtained on a limited sampling of a single transmission area, are extrapolated on a broader scale. We used a modified Ross-Macdonald model with additional compartments for Wolbachia-infected mosquitoes, which differ in their susceptibility to malaria (Fig. 3a) and in the length of their gonotrophic cycle (Fig. 2d), an effect that would likely impact their biting rates (Supplementary Fig. 2). We considered the Wolbachia-infected populations to have an identical mortality rate to uninfected mosquitoes (Fig. 3b, red line) but also ran a model where the increased speed of the gonotrophic cycle is paralleled by a decrease in female lifespan (Fig. 3b, blue line), as predicted by life history theory. Our model suggests that in both scenarios, infection with these bacteria might decrease malaria prevalence in humans, even within the range of Wolbachia frequencies that we observed in mosquito populations.

\section{Discussion}

Our findings that natural Wolbachia infections persist in Anopheles mosquito populations and are negatively correlated to Plasmodium parasite development bring into sharp focus the potential impact of these bacteria on malaria transmission. Although our study is limited to samples from the VK5 village and we have not yet determined the effects of $w$ Anga on malaria transmission by other important vectors like A. gambiae and A. arabiensis, the observed negative correlation between Plasmodium and Wolbachia prevalence in A. coluzzii suggests that malaria dynamics may be affected by the stable presence of these endosymbionts. Indeed, provided our results are confirmed when additional females, species and geographical locations are tested, our mathematical model predicts that increasing 
a

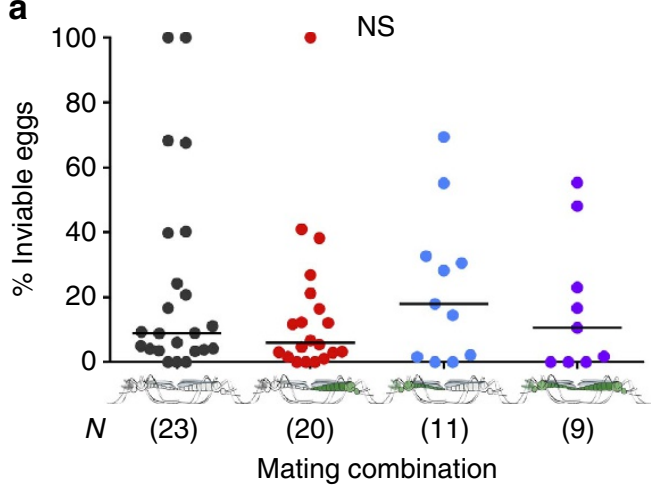

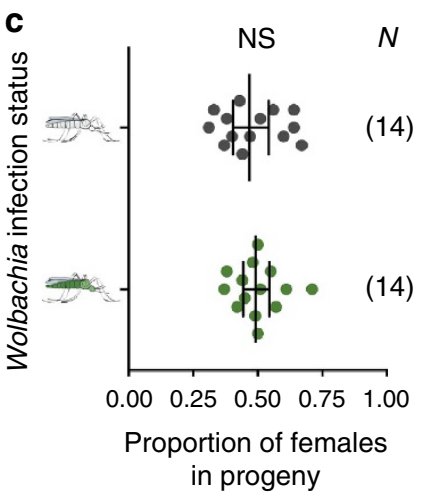

d

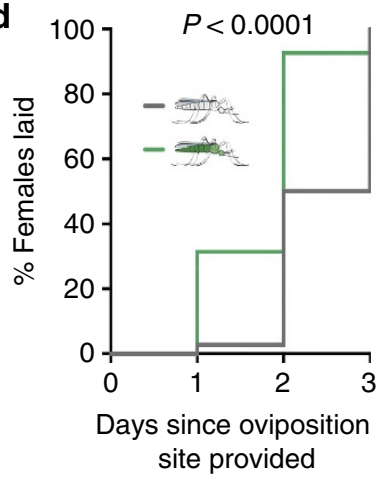

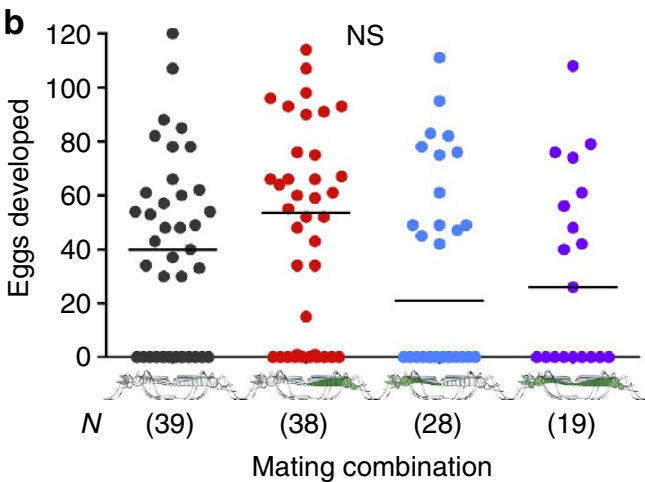

Figure 2 | Analysis of reproductive phenotypes induced by wAnga. (a-c) A. coluzzii females and males collected as eggs or larvae from Vallée du Kou were bred to adulthood and forced mated, and after blood feeding the reproductive output of females was individually scored. wAnga-infected (green) and uninfected (grey) females (right) and males (left) were identified by 165 nested PCR post hoc, and mating couples were divided into four groups (wAnganegative couples, dark grey; wAnga-positive couples, purple; wAnga-positive females mated to wAnga-negative males, red; and wAnga-negative females mated to wAnga-positive males, blue). (a) Egg inviability was calculated by dividing the number of infertile and unhatched eggs in each brood by the total number of eggs 4 days after egg laying. $\mathrm{Cl}$, which would manifest as higher inviability in the cross between wAnga-negative females and wAnga-positive males, was not observed (Kruskal-Wallis test, $\chi^{2}=1.25$, d.f. $=3, P>0.05$ ). (b) The total number of eggs produced by each female was determined by adding the number of eggs laid to the number of eggs remaining in the ovaries at the time of dissection (Kruskal-Wallis, $\chi^{2}=4.09$, d.f. $=3, P>0.05$ ). (c) Hatched larvae from each brood in a were raised to adulthood and sex was scored. The sex ratio of each brood was calculated as the number of female progeny divided by the total progeny (Student's $t$-test on $\log _{10}$-transformed ratios, $t=0.257$, d.f. $=26, P>0.05$ ). (d) A total of 221 blood-fed females were collected from the walls of houses in Vallée du Kou, and 2 days after collection placed in individual oviposition containers to record the timing of egg-laying. The cumulative proportion of females that laid $(n=143)$ on each of 3 consecutive nights following access to an oviposition site is plotted. wAnga-infected females (green line) laid eggs more quickly than uninfected females (grey line) (log-rank test, $\chi^{2}=32.36$, d.f. $=1, P<0.0001$ ). In $\mathbf{a}$ and $\mathbf{b}$, horizontal lines represent the medians. In c, vertical lines and error bars represent the geometric mean and the $95 \%$ confidence intervals, respectively. Numbers in parentheses indicate the sample size.

Wolbachia prevalence would reduce malaria infections in the human population, even when incorporating an increase in biting rates and regardless of a possible trade-off in lifespan. How can $w$ Anga infections have such a significant impact on Plasmodium transmission? A number of studies have shown that strong antipathogenic effects likely caused by immune system activation are often associated with novel Wolbachia transinfections ${ }^{14-16,19}$. Consistent with the previously determined imperfect maternal transmission rates ${ }^{23}$ and the lack of CI detected in this study, the observed interference with Plasmodium suggests that wAnga represents a recently introduced infection not yet fully adapted to the Anopheles host. Although we did not characterize localization of these bacteria in tissues besides the ovaries, we previously detected $w$ Anga in A. coluzzii carcasses that excluded germline tissues $^{23}$. These results suggest $w$ Anga also infects somatic tissues, including those where Plasmodium development occurs, possibly inducing parasite killing via the upregulation of the mosquito immune system.

As hypothesized in other Wolbachia infections ${ }^{16,31-33}$, wAnga may also effectively compete with Plasmodium for nutrient resources. Following blood feeding, large amounts of lipid transporters circulate to transfer lipids from the gut and the fat body to the ovary. These same lipid transporters are required by Plasmodium to evade the mosquito immune system ${ }^{34,35}$. Wolbachia's potential diversion of resources may allow increased rates of immune system-mediated killing of Plasmodium. Alternatively, these endosymbionts may compete with other bacteria in the microbiome, especially following a blood meal ${ }^{36}$, further draining resources away from developing Plasmodium parasites. Regardless of the mechanism, in future studies it will be crucial to test Wolbachia-Plasmodium interactions in different geographical locations and across different transmission seasons to determine the possible heterogeneity in pathogen-blocking effects across Sub-Saharan Africa, a factor that would have consequences for the interpretation of the outcome of key malaria control strategies such as LLINs and indoor residual sprays ${ }^{3}$.

We found that $w$ Anga infections cause limited reproductive phenotypes in the $A$. coluzzii host, and in our mating assays we did not detect CI at significant levels. Although observed in multiple Wolbachia-infected arthropods, CI is not a universal phenomenon, and its presence and penetrance is highly dependent on Wolbachia strain and host organism 

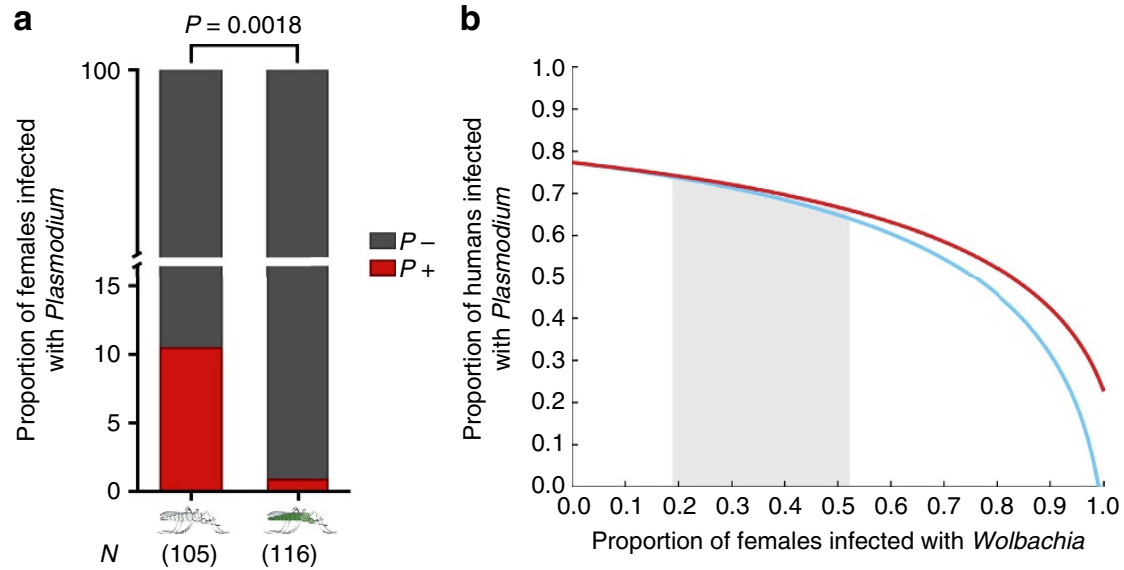

Wolbachia infection status

Figure 3 | wAnga infections reduce malaria prevalence in mosquito populations and in models of human populations. (a) Blood-fed females were collected from houses in Vallée du Kou, and allowed to develop eggs and oviposit. A minimum of 5 days post collection, thoraxes and abdomens were dissected and screened for the presence of wAnga and Plasmodium by $16 \mathrm{~S}$ (or $16 \mathrm{~S}$ nested) PCR and $18 \mathrm{~S}$ quantitative PCR, respectively. Shown in red are the proportions of Plasmodium-infected females in both wAnga-infected (green, $N=116$ ) and uninfected (grey, $N=105$ ) females (Fisher's exact post hoc test on unnormalized data, two-tailed, $P=0.0018$; Supplementary Table 2). Numbers in parentheses indicate the sample size. (b) A modified Ross-Macdonald model incorporating Wolbachia-infected mosquito compartments was used to determine the impact of Wolbachia on malaria prevalence. In addition to variation in biting rate and susceptibility to malaria, Wolbachia-infected mosquitoes were either considered to have an identical daily mortality rate to uninfected mosquitoes (red line) or an enhanced daily mortality rate due to trade-offs with increased gonotrophic cycles (blue line). The shaded area represents the range in Wolbachia prevalence detected in our studies across multiple years.

(reviewed in ref. 37). It remains to be established whether CI could be triggered by $w$ Anga infection in species that do not naturally harbour these endosymbionts, as this would be an important first step towards integrating the use of wAnga into malaria control strategies. A host-dependent ability of Wolbachia to induce CI in non-natural hosts ${ }^{37}$ has been demonstrated in many insects including the major dengue vector Aedes aegypti, in which the fruit fly $w$ Mel strain induces strong $\mathrm{CI}^{38}$ and has successfully invaded natural populations ${ }^{39}$. Furthermore, Wolbachia densities, CI and other reproductive phenotypes can also be modulated within the same host by physiological, ecological and environmental factors including the age of the insect $^{40}$, temperature ${ }^{41}$ and larval density ${ }^{42}$.

Although $w$ Anga did not increase the number of eggs laid by infected females, it increased the rate at which these eggs were laid. Over multiple gonotrophic cycles, this reproductive phenotype could increase lifetime female fecundity, although life history theory predicts that this effect would be counteracted by a trade-off with longevity ${ }^{29}$. The mechanism of this effect may relate to more efficient blood meal digestion, lipid deposition within the egg, egg maturation, activation of hormonal pathways that trigger oviposition ${ }^{43}$ or a combination of these factors. Furthermore, in conditions of nutrient deprivation, $w$ Anga could be provisioning blood-fed females with additional nutrients as shown in other Wolbachia infection models ${ }^{44-46}$, ensuring timely oviposition. These hypotheses remain to be tested, and the colonized Wolbachia-infected A. coluzzii line may prove instrumental to determining the full extent of reproductive manipulations that $w$ Anga inflicts on its mosquito host.

Combined with the previously reported identification in A. gambiae in the same region, the finding of $w$ Anga infections in natural populations of $A$. arabiensis, a vector species capable of remarkable plasticity in its blood feeding and resting behaviour, suggests that this natural Wolbachia strain is capable of adapting to different anophelines. $w$ Anga therefore provides a promising new tool for future malaria control strategies aimed at exophilic and exophagic Anopheles species not targeted by current vector control strategies.

\section{Methods}

Mosquito collections. Two Anopheles species were collected from two separate field sites near Bobo-Dioulasso, Burkina Faso. A. coluzzii were collected from the VK5 village $\left(11^{\circ} 23^{\prime} \mathrm{N} ; 4^{\circ} 24^{\prime} \mathrm{W}\right)$ in the Vallée du Kou, located $30 \mathrm{~km}$ northwest of Bobo-Dioulasso. Mosquitoes were collected in two ways: (1) blood-fed adult females were captured in houses in the village; and (2) fourth instar larvae were collected from breeding sites in the rice fields surrounding the village and bred to adulthood in an insectary. A. arabiensis were collected from houses in the village of Soumousso $\left(11^{\circ} 00^{\prime} \mathrm{N} ; 4^{\circ} 02^{\prime} \mathrm{W}\right)$ located $55 \mathrm{~km}$ northeast of Bobo-Dioulasso as blood-fed adult females.

Establishment of a wAnga-infected A. coluzzii colony. wAnga-infected A. coluzzii mosquitoes were colonized by breeding eggs collected from VK5 to adulthood in an insectary. Adult females emerging from these eggs were crossed to A. coluzzii males from the Mopti colony originating from Mali (obtained through BEI Resources, NIAID, NIH: A. gambiae, Strain MOPTI, MRA-763, contributed by Gregory C. Lanzaro) over two generations. At each generation, after egg laying, infection in these hybrids was determined via PCR on Wolbachia 16S rDNA (see section ' $w$ Anga detection by PCR and sequencing'), and the progeny of $w$ Anga-infected mothers was pooled to establish the line. A line with the same genetic background but cleared of wAnga infection was established by treating a subset of the F3 generation with tetracycline during larval development $\left(1 \mu \mathrm{g} \mathrm{ml}^{-1}\right)$ and adulthood $\left(10 \mu \mathrm{g} \mathrm{ml}^{-1}\right)$.

DNA extraction and mosquito species genotyping. Mosquitoes were beheaded and the genomic DNA of carcasses was extracted using the Qiagen Blood and Tissue kit (Qiagen) with an extended lysis incubation step. In brief, carcasses were homogenized in $1 \times$ phosphate-buffered saline (PBS) and incubated with the kit's proteinase $\mathrm{K}$ and $\mathrm{AL}$ lysis buffer for $30 \mathrm{~min}$ at $56^{\circ} \mathrm{C}$. The remaining extraction steps were performed following the kit's supplementary protocol for DNA extraction of insect cells. For A. coluzzii, A. gambiae and A. arabiensis genotyping the $S 200$ X6.1 locus from genomic DNA was amplified by PCR using standard protocols and with the following primers: forward $5^{\prime}$-TCGCCTTAGACCTTGCG TTA-3', reverse 5'-CGCTTCAAGAATTCGAGATAC-3' (ref. 47).

wAnga detection by PCR and sequencing. Detection of $w$ Anga infection in mosquito carcasses was performed by PCR amplification of the 16S rDNA region using Wolbachia-specific primers (W-Specf: 5'-CATACCTATTCGAAGGGA TAG-3', W-Specr: 5'-AGCTTCGAGTGAAACCAATTC-3') and standard protocols ${ }^{48}$. In cases of low infection intensity, nested PCR was used for $w$ Anga detection. A total of $2 \mu \mathrm{l}$ of the amplification product from the initial 16S rDNA PCR was amplified using specific internal primers (16SNF: $5^{\prime}$-GAAGGGATAGG GTCGGTTCG-3'; 16SNR: 5'-CAATTCCCATGGCGTGACG-3') and HotStarTaq (Qiagen) in a $20 \mu \mathrm{l}$ reaction volume. The nested $16 \mathrm{~S}$ rDNA PCR cycling conditions used were $15 \mathrm{~min}$ at $95^{\circ} \mathrm{C}$, followed by 35 cycles of $15 \mathrm{~s}$ at $95^{\circ} \mathrm{C}, 25 \mathrm{~s}$ at $66^{\circ} \mathrm{C}$ and 
$30 \mathrm{~s}$ at $72{ }^{\circ} \mathrm{C}$, followed by $5 \mathrm{~min}$ at $72^{\circ} \mathrm{C}$. The sequence of the resulting 412 -bp fragment was determined by Sanger sequencing. All samples sequenced were confirmed to correspond to Wolbachia (Supplementary Fig. 3), demonstrating that the nested PCR protocol did not generate any false positives.

wAnga detection in mosquito oocytes by FISH. DNA probes specific to $w$ Anga $16 \mathrm{~S}$ rDNA were designed and synthesized (5'-Cy3-CGAGGCTAAGCTAATCCC TTAAA- $3^{\prime}$, Integrated DNA Technologies). The ovaries of $w$ Anga-infected and tetracycline-treated females from the established $w$ Anga colony were dissected in $1 \times$ PBS, fixed in $4 \%$ paraformaldehyde, and treated with $80 \%$ ethanol-6\% hydrogen peroxide to remove autofluorescence. FISH was then conducted using a protocol modified from Toomey et al. ${ }^{49}$. Following initial pre-hybridization equilibration steps, hybridization was conducted by incubating tissues with $1 \mathrm{ng} \mathrm{Hl}^{-1}$ of the RNA probe in hybridization solution containing $50 \%$ formamide, $5 \times$ SSC, $250 \mu \mathrm{g} \mathrm{ml}^{-1}$ salmon sperm DNA, $0.5 \times$ Denhardt's solution, $20 \mathrm{mM}$ Tris- $\mathrm{HCl}$ and $0.1 \%$ Tween at $37^{\circ} \mathrm{C}$ for $3 \mathrm{~h}$. Probe competition hybridization was conducted by incubating tissues with a mixture of the labelled RNA probe $\left(1 \mathrm{ng} \mu \mathrm{l}^{-1}\right)$ and an unlabelled probe of identical sequence $\left(20 \mathrm{ng} \mu \mathrm{l}^{-1}\right)$. Following washes in a solution containing $1 \times \mathrm{SSC}, 0.1 \%$ Tween and $20 \mathrm{mM}$ Tris- $\mathrm{HCl}$ then in a solution with $0.5 \times$ SSC, $0.1 \%$ Tween and $20 \mathrm{mM}$ Tris- $\mathrm{HCl}$ at $55^{\circ} \mathrm{C}$, tissues were blocked in $1 \%$ BSA in $1 \times$ PBS- $0.1 \%$ Tween (PBST) and stained with 1:100 mouse anti-Cy3 antibody (Santa Cruz Biotechnology). Tissues were then incubated in 1:1,000 goat anti-mouse Alexa488 antibody (ThermoFisher Scientific) and in $1 \mu \mathrm{g} \mathrm{ml}^{-1}$ 4,6-diamidino-2-phenylindole (Sigma-Aldrich). All staining steps were followed by washes in PBST. Tissues were then mounted in Vectashield mounting media and images were acquired on a Zeiss Axio Observer inverted fluorescent microscope with Apotome2. Post-imaging processing was done using ImageJ and Adobe Photoshop CS5.

Analysis of reproductive phenotypes. For CI, fecundity and sex-ratio analyses, mosquitoes were collected as fourth instar larvae from breeding sites or as eggs deposited by blood-fed females collected from houses in VK5 in the Vallée du Kou. These field-collected larvae and eggs were bred to adulthood in the insectary, and adult males and females were crossed in single pairings by forced mating (protocol available on https://www.beiresources.org/Publications/MethodsinAnophelesResearch.aspx). DNA from males was immediately extracted and wAnga infection status was determined by $16 \mathrm{~S}$ PCR. Females were blood-fed and placed into individual cups to allow them to oviposit individually. Following oviposition, the genomic DNA of these females was extracted and screened for $w$ Anga infection by $16 \mathrm{~S}$ PCR. Egg broods were scored for inviability (a direct indicator of CI) and the number of eggs laid (fecundity). Females were also dissected to determine the number of eggs retained and consequently the total number of eggs developed. To assess the sex ratio of the progeny, broods from these crosses were bred to adulthood and the number of males and females in each brood was determined. Infection status of males and females in each cross was determined post hoc by 16S PCR.

For timing of oviposition, blood-fed females were collected from houses in VK5 and put into cups 2 days post blood feeding to allow them to oviposit individually. Oviposition time was monitored by checking for the presence of eggs in each cup every day following provision of an oviposition site.

Plasmodium detection by quantitative PCR. The quantitative PCR protocol published by Bass et al. ${ }^{50}$ for Plasmodium detection was modified for use with SYBR green dye. Genomic DNA extracted from female carcasses was 10-fold diluted, and $5 \mu \mathrm{l}$ of this dilution was quantified in a mix with $1 \times$ Fast SYBR Green Master Mix (ThermoFisher Scientific) and $300 \mathrm{nM}$ of each primer targeting a region in the Plasmodium 18S rDNA sequence (PlasF: $5^{\prime}$-CTTAGTTACGATTA ATAGGAGTAGC-3'; PlasR: 5'-GAAAATCTAAGAATTTCACCTCTGA-3') in duplicate reactions on a StepOnePlus Real-Time PCR System (Applied Biosystems). Relative quantities were calculated using a standard curve built with serial dilutions of a plasmid containing the targeted 18S rDNA sequence. This plasmid was made by PCR amplifying the $18 \mathrm{~S}$ rDNA region of genomic DNA extracted from the P. falciparum P2G12 strain with the PlasF and PlasR primers, and cloning the resulting product using the pGEM-T Vector System (Promega). Copy number quantification was determined using a Nanodrop 2000c Spectrophotometer (ThermoFisher Scientific).

Model of feeding time. Individual female mosquitoes were simulated stochastically through the course of their adult life. On emerging as adults, all female mosquitoes rested for 1 night before mating. The following night females took their initial blood meal, beginning gonotrophic cycles, which consisted of feeding, resting for up to 3 nights and egg laying. The number of nights spent resting before egg laying by each female in each gonotrophic cycle was determined probabilistically from the data on oviposition (Fig. 2d). This process was simulated in MATLAB R2015a (The MathWorks, Inc., Natick, MA, USA) for one million mosquitoes to determine what proportion of mosquitoes fed each night.

Malaria transmission model. The Ross-Macdonald compartmental model of malaria transmission ${ }^{51,52}$ was adapted to include Wolbachia-infected mosquitoes, forming a system of five delay differential equations. The infected human population $\left(I_{\mathrm{H}}\right)$ grew when susceptible humans received infectious bites and declined as humans recovered at a fixed rate $(r=0.05)^{51}$. Mosquito populations $\left(E_{\mathrm{M}}\right.$ and $\left.E_{\mathrm{W}}\right)$ became infected after an infectious bite but were not infectious ( $I_{\mathrm{M}}$ and $I_{\mathrm{W}}$ ) until the completion of the latent period, with Wolbachia-infected populations denoted with a $\mathrm{W}$-subscript. The fraction of the mosquito population infected with Wolbachia $(W)$ was constant throughout a single simulation.

$$
\begin{aligned}
\frac{\mathrm{d} I_{\mathrm{H}}(t)}{\mathrm{d} t}= & a b m\left[I_{\mathrm{M}}(t)+I_{\mathrm{W}}(t)\right]\left[1-I_{\mathrm{H}}(t)\right]-r I_{\mathrm{H}}(t) \\
\frac{\mathrm{d} E_{\mathrm{M}}(t)}{\mathrm{d} t}= & a c I_{\mathrm{H}}(t)\left[(1-W)-E_{\mathrm{M}}(t)-I_{\mathrm{M}}(t)\right]- \\
& a c I_{\mathrm{H}}(t-\tau)\left[(1-W)-E_{\mathrm{M}}(t-\tau)-I_{\mathrm{M}}(t-\tau)\right] e^{-u \tau}-u E_{\mathrm{M}}(t) \\
\frac{\mathrm{d} I_{\mathrm{M}}(t)}{\mathrm{d} t}= & a c I_{\mathrm{H}}(t-\tau)\left[(1-W)-E_{\mathrm{M}}(t-\tau)-I_{\mathrm{M}}(t-\tau)\right] e^{-u \tau}-u I_{\mathrm{M}}(t) \\
\frac{\mathrm{d} E_{\mathrm{W}}(t)}{\mathrm{d} t}= & \hat{a} \hat{c} I_{\mathrm{H}}(t)\left[W-E_{\mathrm{W}}(t)-I_{\mathrm{W}}(t)\right]- \\
& \hat{a} \hat{c} I_{\mathrm{H}}(t-\tau)\left[W-E_{\mathrm{W}}(t-\tau)-I_{\mathrm{W}}(t-\tau)\right] e^{-\hat{u} \tau}-\hat{u} E_{\mathrm{W}}(t) \\
\frac{\mathrm{d} I_{\mathrm{W}}(t)}{\mathrm{d} t}= & \hat{a} \hat{c} I_{\mathrm{H}}(t-\tau)\left[W-E_{\mathrm{W}}(t-\tau)-I_{\mathrm{W}}(t-\tau)\right] e^{-\hat{u} \tau}-\hat{u} I_{\mathrm{W}}(t)
\end{aligned}
$$

The Wolbachia-infected and -uninfected mosquitoes differed only in biting rate ( $a=0.5$ and $\hat{a}=0.565)$, susceptibility to malaria infection $(c=0.79$ and $\hat{c}=0.07)$ and daily mortality rate $\left(u=0.15\right.$, red line $e^{53-55}$; and $\hat{u}=0.175$, blue line in Fig. $\left.3 \mathrm{~b}\right)$. The probability of transition to humans $(b=0.4)^{51}$, the length of the latent period $(\tau=12)^{56}$ and the relative mosquito to human ratio $(m=7.7)^{51}$ were identical between both mosquito populations. Hats denote parameters associated with Wolbachia-infected mosquitoes. The biting rate $(\hat{a})$ was enhanced by the relative number of blood feeds possible by Wolbachia-infected females compared with Wolbachia-uninfected females as determined in our feeding model, $\hat{a}=1.13 a$ (Supplementary Fig. 2). The susceptibility of Wolbachia-infected females to malaria infection $(\hat{c})$ was discounted based on the data presented in Fig. $3 \mathrm{a}, \hat{c}=c / 11$. The mortality rate $(\hat{u})$ was increased (Fig. $3 \mathrm{~b}$, blue line) to impose a fitness costs on Wolbachia-infected females, such that the average number of feeds was identical for Wolbachia-infected and uninfected mosquitoes. All simulations were performed in MATLAB R2015a (The MathWorks, Inc., Natick, MA, USA) using the dde 23 solver.

Data availability. The authors declare that the data supporting the findings of this study are available within the article and its Supplementary Information files.

\section{References}

1. WHO. World Malaria Report (World Health Organization, 2014).

2. Harrison, G. Mosquitoes, Malaria, and Man: A History of the Hostilities Since 1880 (John Murray, 1978).

3. Bhatt, S. et al. The effect of malaria control on Plasmodium falciparum in Africa between 2000 and 2015. Nature 526, 207-211 (2015).

4. Hemingway, J. The role of vector control in stopping the transmission of malaria: threats and opportunities. Phil. Trans. R. Soc. B 369, 20130431 (2014).

5. Toé, K. H. et al. Increased pyrethroid resistance in malaria vectors and decreased bed net effectiveness, Burkina Faso. Emerg. Infect. Dis. 20, 1691-1696 (2014).

6. malERA Consultative Group on Vector Control. A research agenda for malaria eradication: vector control. PLoS Med. 8, e1000401 (2011).

7. Bourtzis, K. et al. Harnessing mosquito-Wolbachia symbiosis for vector and disease control. Acta Trop. 132(Suppl): S150-S163 (2014).

8. Werren, J. H., Baldo, L. \& Clark, M. E. Wolbachia: master manipulators of invertebrate biology. Nat. Rev. Microbiol. 6, 741-751 (2008).

9. Ghelelovitch, S. Genetic determinism of sterility in the cross-breeding of various strains of Culex autogenicus Roubaud. C. R. Hebd. Seances Acad. Sci. 234, 2386-2388 (1952).

10. Werren, J. H. Biology of Wolbachia. Annu. Rev. Entomol. 42, 587-609 (1997)

11. Weeks, A. R. \& Breeuwer, J. A. Wolbachia-induced parthenogenesis in a genus of phytophagous mites. Proc. R. Soc. Lond. B 268, 2245-2251 (2001).

12. Negri, I., Pellecchia, M., Mazzoglio, P. J., Patetta, A. \& Alma, A. Feminizing Wolbachia in Zyginidia pullada (Insecta, Hemiptera), a leafhopper with an XX/XO sex-determination system. Proc. R. Soc. B 273, 2409-2416 (2006).

13. Dyer, K. A. \& Jaenike, J. Evolutionary stable infection by a male-killing endosymbiont in Drosophila innubila: molecular evidence from the host and parasite genomes. Genetics 168, 1443-1455 (2004).

14. Bian, G. et al. Wolbachia Invades Anopheles stephensi populations and induces refractoriness to Plasmodium infection. Science 340, 748-751 (2013).

15. Hughes, G. L., Koga, R., Xue, P., Fukatsu, T. \& Rasgon, J. L. Wolbachia infections are virulent and inhibit the human malaria parasite Plasmodium falciparum in Anopheles gambiae. PLoS Pathog. 7, e1002043 (2011).

16. Moreira, L. A. et al. A Wolbachia symbiont in Aedes aegypti limits infection with dengue, chikungunya, and Plasmodium. Cell 139, 1268-1278 (2009). 
17. van den Hurk, A. F. et al. Impact of Wolbachia on infection with chikungunya and yellow fever viruses in the mosquito vector Aedes aegypti. PLoS Negl. Trop. Dis. 6, e1892 (2012).

18. Glaser, R. L. \& Meola, M. A. The native Wolbachia endosymbionts of Drosophila melanogaster and Culex quinquefasciatus increase host resistance to West Nile virus infection. PLoS ONE 5, e11977 (2010).

19. Kambris, Z. et al. Wolbachia stimulates immune gene expression and inhibits plasmodium development in Anopheles gambiae. PLoS Pathog. 6, e1001143 (2010).

20. Bian, G., Xu, Y., Lu, P., Xie, Y. \& Xi, Z. The endosymbiotic bacterium Wolbachia induces resistance to dengue virus in Aedes aegypti. PLoS Pathog. 6, e1000833 (2010)

21. Blagrove, M. S., Arias-Goeta, C., Failloux, A. B. \& Sinkins, S. P. Wolbachia strain wMel induces cytoplasmic incompatibility and blocks dengue transmission in Aedes albopictus. Proc. Natl Acad. Sci. USA 109, 255-260 (2012).

22. Rances, E., Ye, Y. H., Woolfit, M., McGraw, E. A. \& O'neil, S. L. The relative importance of innate immune priming in Wolbachia-mediated dengue interference. PLoS Pathog. 8, e1002548 (2012).

23. Baldini, F. et al. Evidence of natural Wolbachia infections in field populations of Anopheles gambiae. Nat. Commun. 5, 3985 (2014).

24. Kittayapong, P., Baisley, K. J., Baimai, V. \& O’Neill, S. L. Distribution and diversity of Wolbachia infections in Southeast Asian mosquitoes (Diptera: Culicidae). J. Med. Entomol. 37, 340-345 (2000).

25. Ricci, I., Cancrini, G., Gabrielli, S., D’Amelio, S. \& Favi, G. Searching for Wolbachia (Rickettsiales: Rickettsiaceae) in mosquitoes (Diptera: Culicidae): large polymerase chain reaction survey and new identifications. J. Med. Entomol. 39, 562-567 (2002).

26. Rasgon, J. L. \& Scott, T. W. An initial survey for Wolbachia (Rickettsiales: Rickettsiaceae) infections in selected California mosquitoes (Diptera: Culicidae). J. Med. Entomol. 41, 255-257 (2004).

27. Buck, M. et al. Bacterial associations reveal spatial population dynamics in Anopheles gambiae mosquitoes. Sci. Rep. 6, 22806 (2016).

28. Rasgon, J. L. Wolbachia induces male-specific mortality in the mosquito Culex pipiens (LIN strain). PLoS ONE 7, e30381 (2012).

29. Harshman, L. G. \& Zera, A. J. The cost of reproduction: the devil in the details. Trends Ecol. Evol. 22, 80-86 (2007).

30. Dabire, R. K. et al. Population dynamics of Anopheles gambiae s.l. in Bobo-Dioulasso city: bionomics, infection rate and susceptibility to insecticides. Parasit Vectors 5, 127 (2012).

31. Osborne, S. E., Leong, Y. S., O’Neill, S. L. \& Johnson, K. N. Variation in antiviral protection mediated by different Wolbachia strains in Drosophila simulans. PLoS Pathog. 5, e1000656 (2009).

32. Osborne, S. E., Iturbe-Ormaetxe, I., Brownlie, J. C., O’Neill, S. L. \& Johnson, K. N. Antiviral protection and the importance of Wolbachia density and tissue tropism in Drosophila simulans. Appl. Environ. Microbiol. 78, 6922-6929 (2012).

33. Lu, P., Bian, G., Pan, X. \& Xi, Z. Wolbachia induces density-dependent inhibition to dengue virus in mosquito cells. PLoS Negl. Trop. Dis. 6, e1754 (2012).

34. Mendes, A. M. et al. Conserved mosquito/parasite interactions affect development of Plasmodium falciparum in Africa. PLoS Pathog. 4, e1000069 (2008).

35. Rono, M. K., Whitten, M. M., Oulad-Abdelghani, M., Levashina, E. A. \& Marois, E. The major yolk protein vitellogenin interferes with the antiplasmodium response in the malaria mosquito Anopheles gambiae. PLoS Biol. 8, e1000434 (2010).

36. Hughes, G. L. et al. Native microbiome impedes vertical transmission of Wolbachia in Anopheles mosquitoes. Proc. Natl Acad. Sci. USA 111 , 12498-12503 (2014)

37. Hoffmann, A. A., Ross, P. A. \& Rašić, G. Wolbachia strains for disease control: ecological and evolutionary considerations. Evol. Appl. 8, 751-768 (2015).

38. Walker, T. et al. The wMel Wolbachia strain blocks dengue and invades caged Aedes aegypti populations. Nature 476, 450-453 (2011).

39. Hoffmann, A. A. et al. Successful establishment of Wolbachia in Aedes populations to suppress dengue transmission. Nature 476, 454-457 (2011).

40. Kittayapong, P., Mongkalangoon, P., Baimai, V. \& O’Neill, S. L. Host age effect and expression of cytoplasmic incompatibility in field populations of Wolbachia-superinfected Aedes albopictus. Heredity (Edinb) 88, 270-274 (2002).

41. Bordenstein, S. R. \& Bordenstein, S. R. Temperature affects the tripartite interactions between bacteriophage WO, Wolbachia, and cytoplasmic incompatibility. PLOS ONE 6, e29106 (2011).

42. Gavotte, L., Mercer, D. R., Stoeckle, J. J. \& Dobson, S. L. Costs and benefits of Wolbachia infection in immature Aedes albopictus depend upon sex and competition level. J. Invertebr. Pathol. 105, 341-346 (2010).

43. Gabrieli, P. et al. Sexual transfer of the steroid hormone 20E induces the postmating switch in Anopheles gambiae. Proc. Natl Acad. Sci. USA 111, 16353-16358 (2014).
44. Moriyama, M., Nikoh, N., Hosokawa, T. \& Fukatsu, T. Riboflavin provisioning underlies Wolbachia's fitness contribution to its insect host. mBio 6, e01732-15 (2015).

45. Unckless, R. L. \& Jaenike, J. Maintenance of a male-killing Wolbachia in Drosophila innubila by male-killing dependent and male-killing independent mechanisms. Evolution 66, 678-689 (2012).

46. Brownlie, J. C. et al. Evidence for metabolic provisioning by a common invertebrate endosymbiont, Wolbachia pipientis, during periods of nutritional stress. PLoS Pathog. 5, el000368 (2009).

47. Santolamazza, F. et al. Insertion polymorphisms of SINE200 retrotransposons within speciation islands of Anopheles gambiae molecular forms. Malar. J. 7, 163 (2008).

48. Werren, J. H. \& Windsor, D. M. Wolbachia infection frequencies in insects: evidence of a global equilibrium? Proc. R. Soc. Lond. B 267, 1277-1285 (2000).

49. Toomey, M. E., Panaram, K., Fast, E. M., Beatty, C. \& Frydman, H. M. Evolutionarily conserved Wolbachia-encoded factors control pattern of stem-cell niche tropism in Drosophila ovaries and favor infection. Proc. Natl Acad. Sci. USA 110, 10788-10793 (2013).

50. Bass, C. et al. PCR-based detection of Plasmodium in Anopheles mosquitoes: a comparison of a new high-throughput assay with existing methods. Malar. J. 7, 177 (2008)

51. Wallace, D. I., Southworth, B. S., Shi, X., Chipman, J. W. \& Githeko, A. K. A comparison of five malaria transmission models: benchmark tests and implications for disease control. Malar. J. 13, 268 (2014).

52. Macdonald, G. The Epidemiology and Control of Malaria (Oxford Univ. Press, 1957).

53. Midega, J. T. et al. Estimating dispersal and survival of Anopheles gambiae and Anopheles funestus along the Kenyan coast by using mark-release-recapture methods. J. Med. Entomol. 44, 923-929 (2007).

54. Charlwood, J. D. et al. 'A mate or a meal'-pre-gravid behaviour of female Anopheles gambiae from the islands of Sao Tome and Principe, West Africa. Malar. J. 2, 9 (2003).

55. Costantini, C. et al. Density, survival and dispersal of Anopheles gambiae complex mosquitoes in a west African Sudan savanna village. Med. Vet. Entomol. 10, 203-219 (1996).

56. Baton, L. A. \& Ranford-Cartwright, L. C. Spreading the seeds of millionmurdering death: metamorphoses of malaria in the mosquito. Trends Parasitol. 21, 573-580 (2005)

\section{Acknowledgements}

We thank David Clarke for help with mosquito procedures, Manuela Bernard for assistance with artwork, and Matthias Marti and Deepali Ravel for providing Plasmodium falciparum P2G12 genomic DNA. Research reported in this publication was supported by the National Institute Of Allergy And Infectious Diseases of the National Institutes of Health under Award Number R21AI117313 to FC. This material is based on work supported by the National Science Foundation Graduate Research Fellowship Program under Grant No. DGE1144152 to P.M. Travel to Burkina Faso was in part supported by Harvard University's 'Defeating Malaria: From the Genes to the Globe' Initiative to W.R.S.

\section{Author contributions}

F.C., W.R.S. and P.M. designed the experiments; W.R.S., P.M. and F.B. performed the experiments; F.C., W.R.S. and P.M. analysed the data; L.M.C. and C.O.B. mathematically modelled the data; S.S., R.K.D. and A.D. provided samples for the analysis; F.C., W.R.S P.M. and L.M.C. wrote the manuscript; W.R.S. and P.M. contributed equally to this study. The content is solely the responsibility of the authors and does not necessarily represent the official views of the National Institutes of Health.

\section{Additional information}

Supplementary Information accompanies this paper at http://www.nature.com/ naturecommunications

Competing financial interests: The authors declare no competing financial interests.

Reprints and permission information is available online at http://npg.nature.com/ reprintsandpermissions/

How to cite this article: Shaw, W. R. et al. Wolbachia infections in natural Anopheles populations affect egg laying and negatively correlate with Plasmodium development. Nat. Commun. 7:11772 doi: 10.1038/ncomms11772 (2016).

This work is licensed under a Creative Commons Attribution 4.0 International License. The images or other third party material in this article are included in the article's Creative Commons license, unless indicated otherwise in the credit line; if the material is not included under the Creative Commons license, users will need to obtain permission from the license holder to reproduce the material. To view a copy of this license, visit http://creativecommons.org/licenses/by/4.0/ 